Sādhanā Vol. 37, Part 1, February 2012, pp. 149-169. (C) Indian Academy of Sciences

\title{
Quasi-static crack tip fields in rate-sensitive FCC single crystals
}

\author{
P BISWAS $^{1}$ and R NARASIMHAN ${ }^{2, *}$ \\ ${ }^{1}$ Global General Motors R\&D, India Science Lab, GM Technical Centre (India), \\ Bangalore 560 066, India \\ ${ }^{2}$ Department of Mechanical Engineering, Indian Institute of Science, \\ Bangalore 560012 , India \\ e-mail: narasi@mecheng.iisc.ernet.in
}

\begin{abstract}
In this work, the effects of loading rate, material rate sensitivity and constraint level on quasi-static crack tip fields in a FCC single crystal are studied. Finite element simulations are performed within a mode I, plane strain modified boundary layer framework by prescribing the two term $(K-T)$ elastic crack tip field as remote boundary conditions. The material is assumed to obey a rate-dependent crystal plasticity theory. The orientation of the single crystal is chosen so that the crack surface coincides with the crystallographic (010) plane and the crack front lies along [10 $\overline{1}]$ direction. Solutions corresponding to different stress intensity rates $\dot{K}, T$-stress values and strain rate exponents $m$ are obtained. The results show that the stress levels ahead of the crack tip increase with $\dot{K}$ which is accompanied by gradual shrinking of the plastic zone size. However, the nature of the shear band patterns around the crack tip is not affected by the loading rate. Further, it is found that while positive $T$-stress enhances the opening and hydrostatic stress levels ahead of crack tip, they are considerably reduced with imposition of negative $T$-stress. Also, negative $T$-stress promotes formation of shear bands in the forward sector ahead of the crack tip and suppresses them behind the tip.
\end{abstract}

Keywords. Rate sensitivity; crack tip constraint; quasi-static fields; crystal plasticity; finite elements.

\section{Introduction}

The stress and plastic strain fields prevailing near a stationary crack tip in an elastic-plastic solid govern the crack initiation process. Thus, cleavage crack initiation is determined by the level of opening stress ahead of a tip (Ritchie et al 1973). On the other hand, ductile fracture processes like micro-void growth and coalescence in the process zone ahead of the tip are controlled by the level of plastic strain and hydrostatic stress (Thomason 1990). It is found from recent studies on fatigue loading of polycrystalline alloys that crack initiation and subsequent transgranular crack

*For correspondence 
growth are determined by the plastic slip associated with individual slip systems (Zhai et al 2000; Duber et al 2006; Blochwitz et al 2008). Thus, a thorough understanding of crack tip fields and the influence of various factors such as fracture geometry, material parameters, loading rate and temperature are crucial to undertake fracture mechanics-based design as well as for developing materials which are resistant to fracture. In the context of automobile applications, this will help in improving formability of materials such as aluminum and magnesium alloys.

A comprehensive review of stationary crack tip fields in elastic-plastic solids was recently performed by Narasimhan et al (2009). It is clear from this review that these fields in rateindependent, isotropic plastic solids are now well-understood. The asymptotic structure of these fields in power law hardening plastic solids obeying the von Mises yield condition was first studied by Hutchinson (1968) and Rice \& Rosengren (1968). Their asymptotic solution (referred to as the HRR solution) underscored the unique role played by the $J$-integral proposed by Rice (1968) in controlling the stress and plastic strain variations near the tip. It also showed that high opening and hydrostatic stresses prevail ahead of the tip under mode I plane strain conditions, which aid both cleavage and ductile fracture mechanisms. However, in order to employ $J$ as a single fracture characterizing parameter in ductile materials, it is essential that the $J$-dominant HRR fields should prevail over sufficiently large length scales (Hutchinson 1983).

The limitation of the single parameter characterization by $J$ was demonstrated by McMeeking \& Parks (1979), Betegon \& Hancock (1991) and O'Dowd \& Shih (1991). These studies showed that the level of stress triaxiality (or crack tip constraint) prevailing ahead of the tip under Mode I loading is strongly dependent on the fracture geometry. Thus, while fracture geometries which experience predominantly bending over the uncracked ligament (such as deeply cracked Three Point Bend or TPB specimen) display high constraint (comparable to the levels predicted by the HRR solution), tension dominated geometries such as Centre Crack Panel (or CCP) show much reduced triaxiality levels. This has led to development of two-parameter $J-Q$ description of crack tip fields as well as the fracture resistance in isotropic plastic solids (O'Dowd \& Shih 1991; Anderson 2005). Here, $Q$ is a triaxiality parameter which is close to zero or slightly positive for high constraint geometries and significantly negative for low constraint ones. In order to define $Q$, a difference stress field is constructed between the stress distribution actually prevailing near the tip and a reference solution (O'Dowd \& Shih 1991). The reference field may be chosen as the HRR solution. Alternately, the small-scale yielding solution obtained from a boundary layer analysis setting the $T$-stress, which is the second term in the elastic crack field (Williams 1957), as zero in the remote region far away from the crack tip can be used (O'Dowd \& Shih 1994).

Koppenhoefer \& Dodds (1996), Basu \& Narasimhan (2000) and Jayadevan et al (2002a, b) examined constraint loss under dynamic loading in isotropic solids taking into account the combined effects of rate sensitivity and material inertia. However, it must be noted that HRR solution or the rate-independent small-scale yielding solution with $T=0$ (O’Dowd \& Shih 1994) are not valid reference fields for defining the constraint parameter $Q$ in rate-dependent solids. As pointed out by Basu \& Narasimhan (2000), use of these solutions in the context of rate-dependent materials (as in the work of Koppenhoefer \& Dodds (1996)) will lead to erroneous interpretation of the results. To resolve this issue, Basu \& Narasimhan (2000) employed a modified HRR field obtained by suitably scaling the yield strength based on the strain rate prevailing near the tip. However, such a modification is ad-hoc in nature. Hence, Jayadevan et al (2002b) performed systematic quasi-static analysis of a stationary crack in a rate-sensitive (power law viscous) isotropic plastic solid under small scale yielding conditions with different stress intensity rates $\dot{K}$. They concluded that the near-tip stress and plastic strain fields are characterized by the ratio $\dot{K} /\left(K \dot{\epsilon}_{o}\right)$ in addition to other parameters such as $J$ and $T$. Here $\dot{\epsilon}_{o}$ is a reference strain rate. However, the exact nature of the dependence of these fields for isotropic plastic solids on the 
parameter $\dot{K} /\left(K \dot{\epsilon}_{o}\right)$ was not discerned in their work. Based on the above important observation, Jayadevan et al (2002b) employed the small scale yielding solution obtained with $T=0$ and the respective $\dot{K} /\left(K \dot{\epsilon}_{o}\right)$ value as the reference field to define the constraint parameter $Q$.

It must be emphasized that all the above studies pertain to isotropic plastic solids obeying the von Mises yield condition. However, in reality, most alloys used in engineering applications are polycrystalline in nature. In certain special situations such as high pressure turbine blades of aircraft engines, single crystals of Ni-based superalloys are used. In such cases, the role of material microstructure, dislocation based hardening mechanisms, and lattice orientation of the grains surrounding the tip with respect to the crack plane and loading direction will influence the mechanics of fracture (Crone et al 2004; Patil et al 2010; Blochwitz et al 2008). Further, as already mentioned, the nature of the slip bands occurring in the individual grains surrounding the tip may dictate the fracture behaviour in polycrystalline alloys (Zhai et al 2000; Duber et al 2006; Blochwitz et al 2008). Thus, an understanding of this behaviour from a fundamental perspective can be obtained by examining crack tip fields in single crystals.

Rice (1987) first constructed an asymptotic solution for a stationary crack in rate-independent, ideally plastic FCC single crystal. He considered the specific orientation in which the crack plane coincides with the (010) crystallographic plane and the crack front along the [101] direction which has been frequently observed to occur in experimental investigations (Garrett \& Knott 1975; Neumann 1974). His solution structure involves constant stress sectors separated by discontinuities which correspond to bands of intense shear deformation. In particular, for the above lattice orientation, he predicted slip shear bands at $55^{\circ}$ and $125^{\circ}$ to the crack line which are aligned parallel to the traces of two families of slip lines on the plane of deformation. In addition, a kink shear band at $90^{\circ}$ to the crack line was predicted which is perpendicular to the trace of a third family of slip lines. A recent experimental investigation using Electron Back Scattered Diffraction (EBSD) by Patil et al (2009b) confirmed the existence of the kink shear band at $90^{\circ}$ to a notch surface in TPB specimens of aluminum single crystal. However, previous experimental studies on copper and copper-beryllium single crystals using optical metallography and Moire interferometry technique (e.g., Crone et al 2004) could not detect this band.

Patil et al (2008b, 2010) showed from modified boundary layer analysis that a valid $J-Q$ field exists in rate-independent FCC single crystal irrespective of lattice orientation. They established correlations between the constraint parameter $Q$ and the $T$-stress, with negative values of $T$ inducing significant constraint loss as in isotropic plastic solids. The slip band pattern near the tip was also found to depend on the constraint level. Guided by these numerical results, Patil et al (2009a) constructed two families of slip line solutions in ideally plastic FCC single crystals which display a range of constraint levels. An important feature of these solutions is that they incorporate an elastic sector in the near-tip region. In this connection, it must be mentioned that studies by Arakere et al $(2005,2009)$ in FCC single crystals of superalloys have highlighted the importance of elastic anisotropy on the crack tip fields.

It is clear from the above review that stationary crack tip fields in rate-independent FCC single crystals are now well-understood. On the other hand, there has been no investigation on the effect of strain rate sensitivity and loading rate on the stress and plastic slip distribution as well as constraint levels near a tip. Thus, the objective of this work is to examine the general structure of these distributions and the plastic zone shapes in a rate-sensitive FCC single crystal. To this end, modified boundary layer simulations are conducted assuming a power-law viscous response for the single crystal. The lattice orientation analysed by Rice (1987) is considered. Finite element solutions are generated corresponding to different values of $T$ stress, stress intensity rate $\dot{K}$ and rate exponent $m$. It is found that the near-tip stress distribution scales with the parame$\operatorname{ter}\left(\dot{K} /\left(K \dot{\gamma}_{o}\right)\right)^{m}$ except at very high values of $\dot{K} /\left(K \dot{\gamma}_{o}\right)$, when radial distance from the tip is 
normalized by $J / \tau_{o}$. Here $\tau_{o}$ is the initial slip resistance and $\dot{\gamma}_{o}$ is a reference plastic slip rate. A valid two-parameter $J-Q$ characterization of the crack tip fields is shown to exist when $Q$ is defined using a reference solution with $T=0$ and the same level of $\dot{K} /\left(K \dot{\gamma}_{o}\right)$. Further, while increase in loading rate does not affect the qualitative nature of the plastic slip distribution, but only shrinks the size of the plastic zone at a given value of $K$, constraint level changes the pattern of the shear bands as in rate-independent single crystal.

\section{Constitutive equations}

In this work, the single crystal plasticity model proposed by Asaro (1983) is employed with the assumption of multiplicative decomposition of the deformation gradient tensor $\mathbf{F}$ given by

$$
\mathbf{F}=\mathbf{F}^{*} \mathbf{F}^{p}
$$

In the above equation, $\mathbf{F}^{*}$ constitutes the elastic stretching and rotation part of the deformation gradient tensor, while $\mathbf{F}^{p}$ represents plastic part due to slip in the slip systems. With the assumption of small elastic strains, the Jaumann rate of Kirchhoff stress based on lattice rotation is related to the elastic part of rate of deformation tensor through the elasticity tensor.

The evolution of $\mathbf{F}^{p}$ is related to the activity on individual slip systems and is given by

$$
\mathbf{L}^{p}=\dot{\mathbf{F}}^{p} \mathbf{F}^{p-1}=\sum_{\alpha=1}^{n} \dot{\gamma}_{\alpha} \mathbf{m}^{\alpha}
$$

where $n$, is the number of slip systems while $\dot{\gamma}_{\alpha}$ and $m^{\alpha}$ represents plastic slip rate and plastic flow tensor or Schmid tensor, respectively, on slip system $\alpha$. For a slip system $\alpha$ with slip direction $\mathbf{S}^{\alpha}$ and slip plane normal $\mathbf{N}^{\alpha}$, the Schmid tensor is given by

$$
\mathbf{m}^{\alpha}=\mathbf{S}^{\alpha} \otimes \mathbf{N}^{\alpha}
$$

As suggested by Peirce et al (1983) and Cuitino \& Ortiz (1992), the material rate sensitivity is incorporated by assuming a power-law viscous form of the constitutive response which is given by

$$
\dot{\gamma}_{\alpha}=\dot{\gamma}_{o}\left(\frac{\tau_{\alpha}}{\tau_{\alpha}^{p}}\right)^{\frac{1}{m}}
$$

In the above equation, $\tau_{\alpha}$ and $\tau_{\alpha}^{p}$ represent the resolved shear stress and current strength of slip system $\alpha$, respectively, whereas $\dot{\gamma}_{0}$ is a reference strain rate and $m$ is the strain rate exponent. The single crystal is assumed to be elastic-perfectly plastic so that $\tau_{\alpha}^{p} \equiv \tau_{0}$, where $\tau_{0}$ is the initial slip resistance. The initial slip resistance is generally taken to be same for all slip systems. This is because during initial stages of plastic deformation, barrier to dislocation movement is same for all slip systems. As deformation progresses, the slip resistance may evolve differently on various slip systems for a strain hardening single crystal. In this work, the values of $\dot{\gamma}_{0}, \tau_{0}$, Young's modulus $E$ and Poisson's ratio $v$ are taken as $0.1,10 \mathrm{MPa}, 70 \mathrm{GPa}$ and 0.3 , respectively. These values correspond to aluminum single crystals (Patil et al 2008a). Further, for simplicity, the material is assumed to be elastically isotropic. This assumption is reasonably appropriate for aluminum which does not exhibit pronounced elastic anisotropy. 
The above constitutive equations are implemented in a general purpose finite element code FEAP (Zienkiewicz \& Taylor 1989) and a rate tangent method is used to update the stresses and internal variables (Peirce et al 1983).

\section{Finite element model}

In the modified boundary layer analysis, a large semicircular domain containing a crack along one of its radii is modelled with 2D plane strain finite elements as shown in figure 1a. The radius of the outer boundary is taken sufficiently large so that the plastic deformation is well-contained
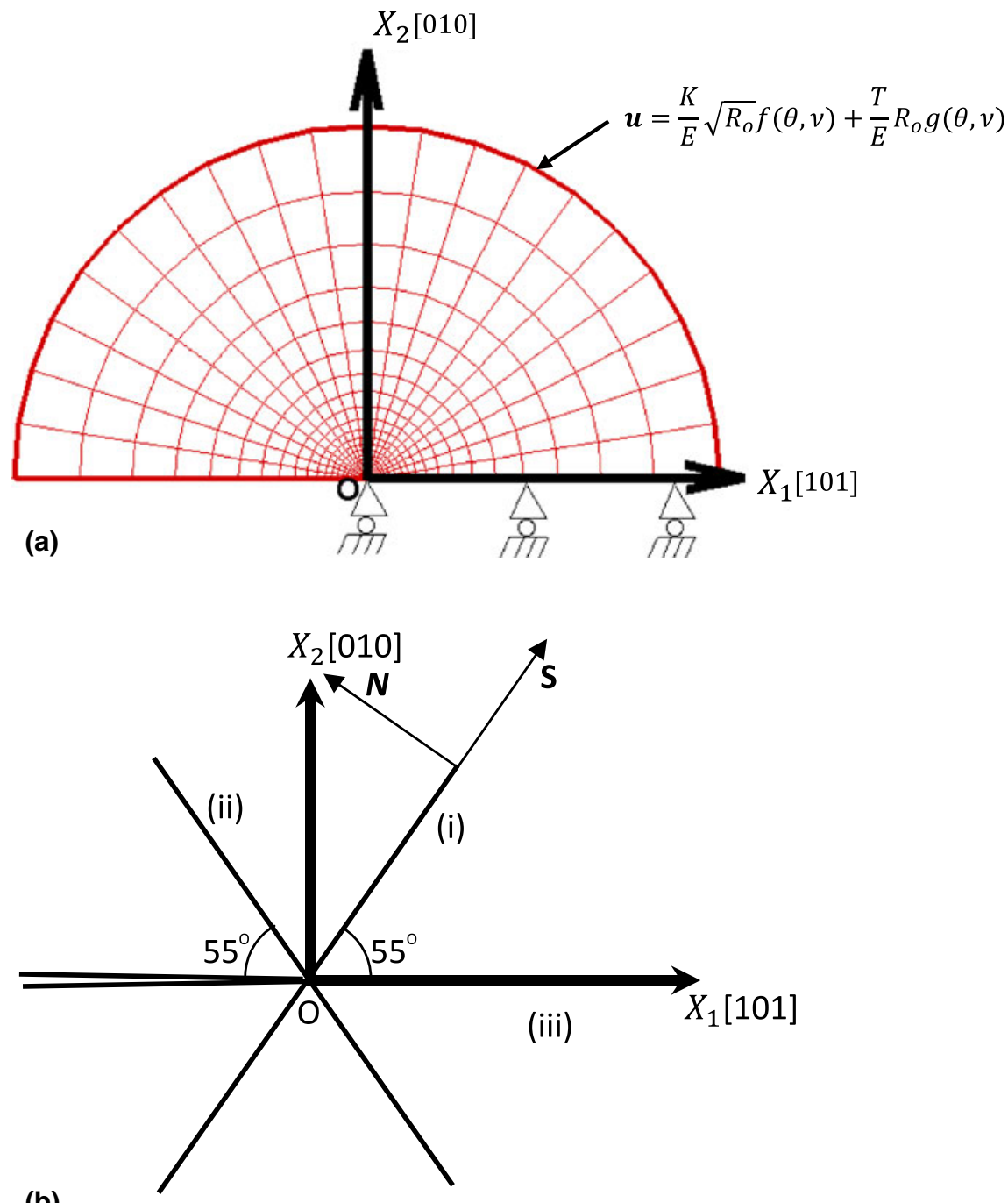

Figure 1. (a) Finite element mesh and boundary conditions along with coordinate system. Due to mode I and crystallographic symmetry, only half of the geometry is modelled with symmetry conditions applied on $X_{2}=0$ line. (b) Traces of slip systems on the plane of deformation. 
within the domain and small scale yielding conditions are preserved. Mode I symmetry boundary conditions are imposed on the line ahead of the crack tip, while traction free boundary conditions are maintained on the crack face. Displacement components based on first and second terms (which are governed by $K$ and $T$, respectively) of linear elastic mode I crack tip field (Williams 1957) are prescribed on the outer boundary as indicated in figure 1a. Here $(r, \theta)$ are polar coordinates centered at the crack tip. It must be mentioned here that the effect of the specimen geometry on the plastic crack tip fields under small to intermediate scale yielding conditions is manifested through the $T$-stress as discussed elaborately by O'Dowd \& Shih $(1991,1992)$ and Betegon \& Hancock (1991) for isotropic rate-independent plastic solids. It must be mentioned that the selected single crystal orientation (the crack plane coinciding with the (010) plane and crack front lying along [101] direction) is also symmetric with respect to the crack plane. With this particular orientation, only three pairs of slip systems can be active to result in plane strain deformation in the $X_{1}-X_{2}$ plane depicted in figure 1a (Rice 1987). These three pairs of slip

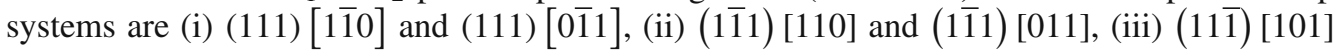
and (111) [101]. Further, each system of a particular pair should have the same plastic slip. It can be shown that the resolved shear stress on each system of a particular pair would indeed be the same, which in view of Eq. (4) ensures that the above condition will hold. Henceforth, these pairs will be referred to as $S_{1}, S_{2}$ and $S_{3}$, respectively. The traces of $S_{1}, S_{2}$ and $S_{3}$ in the plane of deformation are shown in figure $1 \mathrm{~b}$. Static equilibrium solutions for various loading rates are obtained by prescribing displacement rates at the outer boundary corresponding to different $\dot{K}$ while keeping the ratio $T / K$ fixed. The value of $K$ is increased till the desired level of $T$-stress is attained.

\section{Results and discussion}

In this section, results pertaining to the structure of the crack tip field in rate-dependent FCC single crystal will be discussed. In the discussion, the loading rate is described as the prescribed rate of stress intensity factor $\dot{K}$.

\subsection{Effect of loading rate and strain rate exponent $m$}

4.1a Radial variations of stress: Radial variations of normalized stress component $\sigma_{22} / \tau_{o}$ ahead of the crack tip at a fixed value of $K=500 \mathrm{~N}-\mathrm{mm}^{\frac{3}{2}}$ corresponding to different stress intensity rates $\dot{K}$ are shown in figure $2 \mathrm{a}$ and b for $m=0.01$ and 0.1 , respectively. It can be seen from figure 2 a that for the case $m=0.01$, the stress distribution is relatively independent of loading rate indicating that this case corresponds to nearly rate-independent material behaviour. Furthermore, the value of opening stress close to the crack tip (for example, at a normalized distance of of $\left.r /\left(J / \tau_{o}\right)=4\right)$ corroborates well with the fully plastic solution of Rice (1987). On the other hand, for $m=0.1$, the stress level ahead of the crack tip increases strongly with applied loading rate. Thus, at a normalized distance of $r /\left(J / \tau_{o}\right)=4$, the value of $\sigma_{22}$ is $7.1 \tau_{o}$ corresponding to $\dot{K}=10^{4} \mathrm{~N}-\mathrm{mm}^{-\frac{3}{2}} / \mathrm{s}$, whereas it becomes $8.2 \tau_{o}, 8.8 \tau_{o}$ and $10.8 \tau_{o}$ when $\dot{K}$ is increased to $5 \times$ $10^{4}, 10^{5}$ and $10^{6} \mathrm{~N}-\mathrm{mm}^{-\frac{3}{2}} / \mathrm{s}$, respectively. Also, the difference in slope of $\sigma_{22} / \tau_{o}$ with respect to normalized distance $r /\left(J / \tau_{o}\right)$ can be noted between figure $2 \mathrm{a}$ and $\mathrm{b}$. Higher strain rate near the crack tip and larger strain rate sensitivity for $m=0.1$ is responsible for the difference.

The elevation in stress level at higher loading rate can have a strong implication on the fracture mechanism, particularly for the case of brittle (cleavage) fracture. The brittle fracture mechanism 

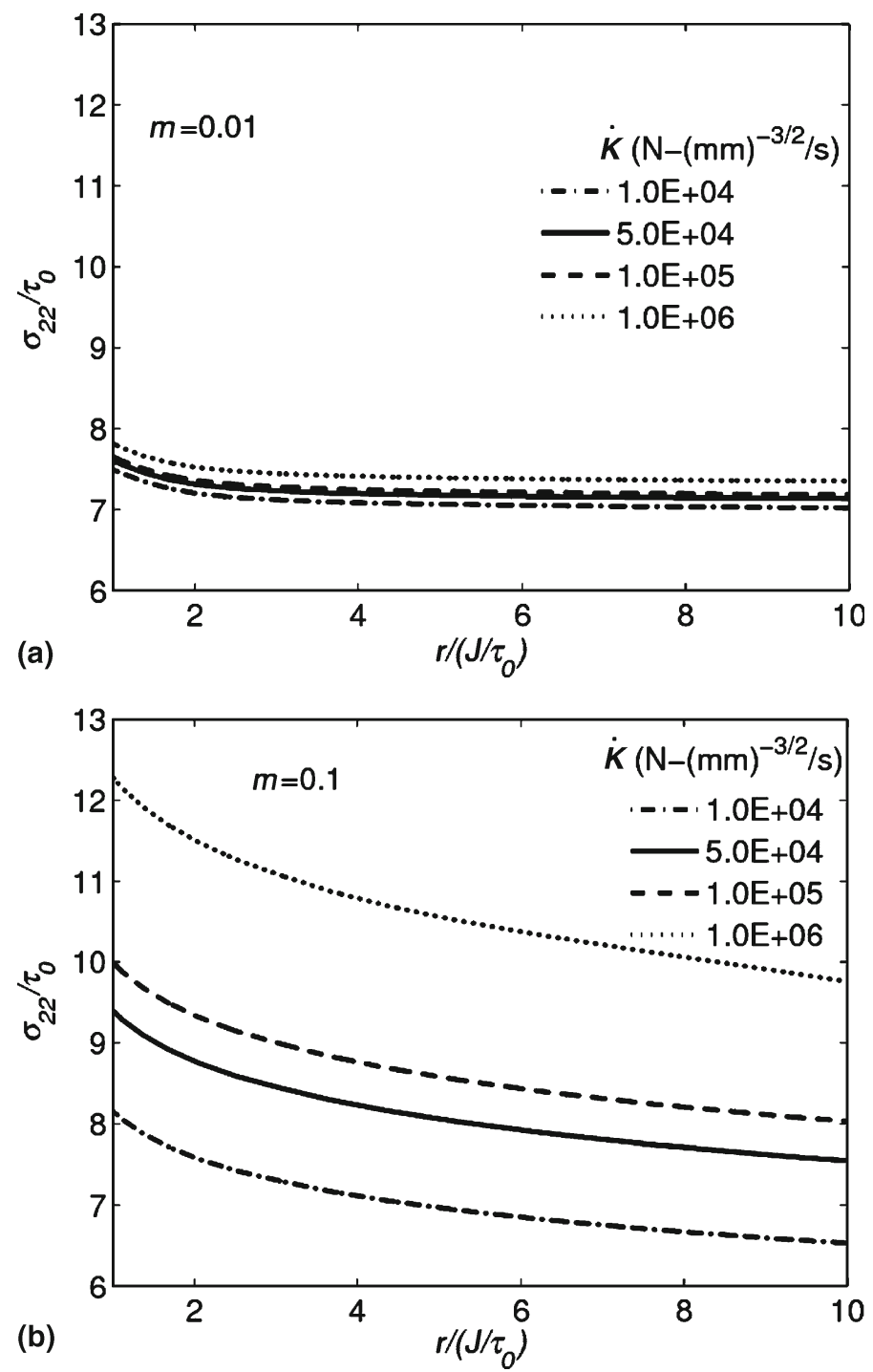

Figure 2. Radial variations of $\sigma_{22} / \tau_{o}$ ahead of the crack tip with normalized distance for different loading rates at a fixed $K$ of $500 \mathrm{~N}-\mathrm{mm}^{-\frac{3}{2}}$ and $T=0$ corresponding to (a) $m=0.01$, (b) $m=0.1$.

is solely governed by the opening stress component (Ritchie et al 1973) and elevated stress level at higher loading rate can accelerate the fracture process.

4.1b Contours of plastic slip: Contour plots of plastic slip in slip systems $S_{1}, S_{2}$ and $S_{3}$ are shown in figure $3 \mathrm{a}, \mathrm{b}$ and $\mathrm{c}$, respectively, at a fixed value of $K=500 \mathrm{~N}-\mathrm{mm}^{-\frac{3}{2}}$ and $\dot{K}=10^{4} \mathrm{~N}-\mathrm{mm}^{-\frac{3}{2}} / \mathrm{s}$ corresponding to $m=0.1$. Similar set of plots are presented in figure $4 \mathrm{a}-\mathrm{c}$ under identical conditions but for $\dot{K}=10^{6} \mathrm{~N}-\mathrm{mm}^{-\frac{3}{2}} / \mathrm{s}$. In all the plots, the same contour levels are displayed to facilitate direct comparison. Also the coordinate axes are normalized by 

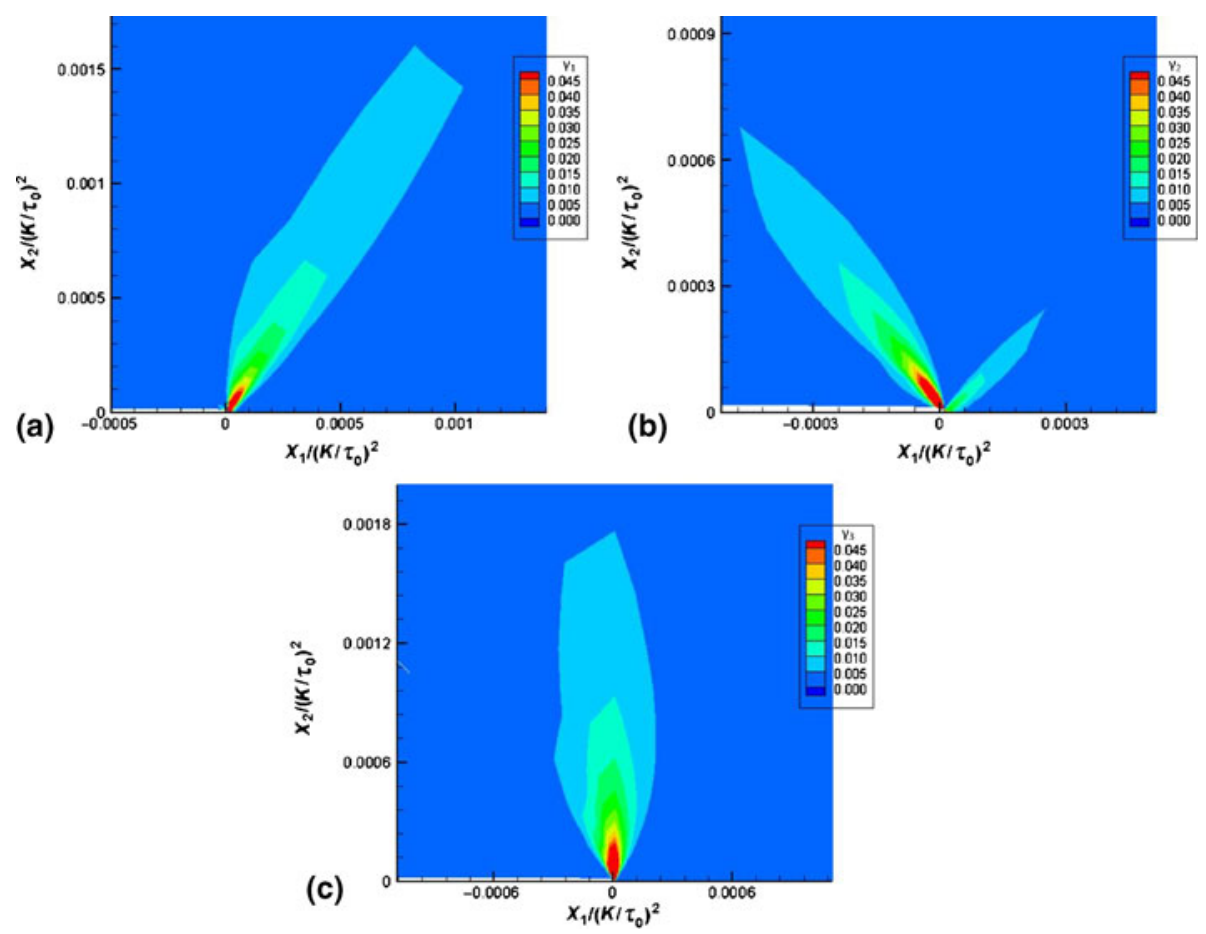

Figure 3. Fringe contours of plastic slip on slip system (a) $S_{1}$, (b) $S_{2}$ and (c) $S_{3}$ at $K=500 \mathrm{~N}-\mathrm{mm}^{-\frac{3}{2}}$, $T=0$ and $\dot{K}=10^{4} \mathrm{~N}-\mathrm{mm}^{-\frac{3}{2}} / \mathrm{s}$ for $m=0.1$.

$\left(K / \tau_{o}\right)^{2}$. It can be seen from figure 3a that the activity on slip system $S_{1}$ produces a strong slip shear band that makes an angle $\theta=55^{\circ}$ with the $X_{1}$ axis. On the other hand, slip activity on $S_{2}$ creates a slip shear band at $\theta=125^{\circ}$ and another small and weak kink shear band at $\theta \approx 45^{\circ}$ (see figure $3 \mathrm{~b}$ ). Figure $3 \mathrm{c}$ shows formation of a strong kink shear band at $\theta=90^{\circ}$ due to activity on slip system $S_{3}$. It must be noted here that a slip shear band is aligned parallel to the slip direction and involves gliding of dislocations emitted from the tip. By contrast, a kink shear band is almost perpendicular to the slip direction and involves expansion of dislocation dipole loops which are formed from internal sources (Rice 1987). The latter gives rise to strong lattice rotation and can be detected in experimental studies using a method such as EBSD (Patil et al 2009b).

On comparing figure $3 \mathrm{a}-\mathrm{c}$ with figure $4 \mathrm{a}-\mathrm{c}$, it can be seen that imposition of higher loading rate does not change the character of the slip bands. However, it diminishes the radial extents of all the bands. Thus, for example, the radial extent of the contour corresponding to $\gamma_{1}=0.005$ is $0.0018\left(K / \tau_{o}\right)^{2}$ for $\dot{K}=10^{4} \mathrm{~N}-\mathrm{mm}^{-\frac{3}{2}} / \mathrm{s}$ (see figure $3 \mathrm{a}$ ). The radial spread of the same contour becomes $0.0012\left(K / \tau_{o}\right)^{2}$ when $\dot{K}=10^{6} \mathrm{~N}-\mathrm{mm}^{-\frac{3}{2}} / \mathrm{s}$ (see figure $4 \mathrm{a}$ ). Indeed, it was found that the slip rate $\dot{\gamma}_{i}$ is higher for higher loading rate. However, longer time duration needed to attain a certain value of $K$ for the case of lower loading rate results in larger total accumulated plastic slip as compared to the case of higher loading rate.

4.1c Contours of maximum principal logarithmic plastic stretch: The contours of maximum principal $\operatorname{logarithmic}$ plastic stretch $\log \lambda_{1}^{p}$, is shown in figure 5 at a fixed $K$ level of 

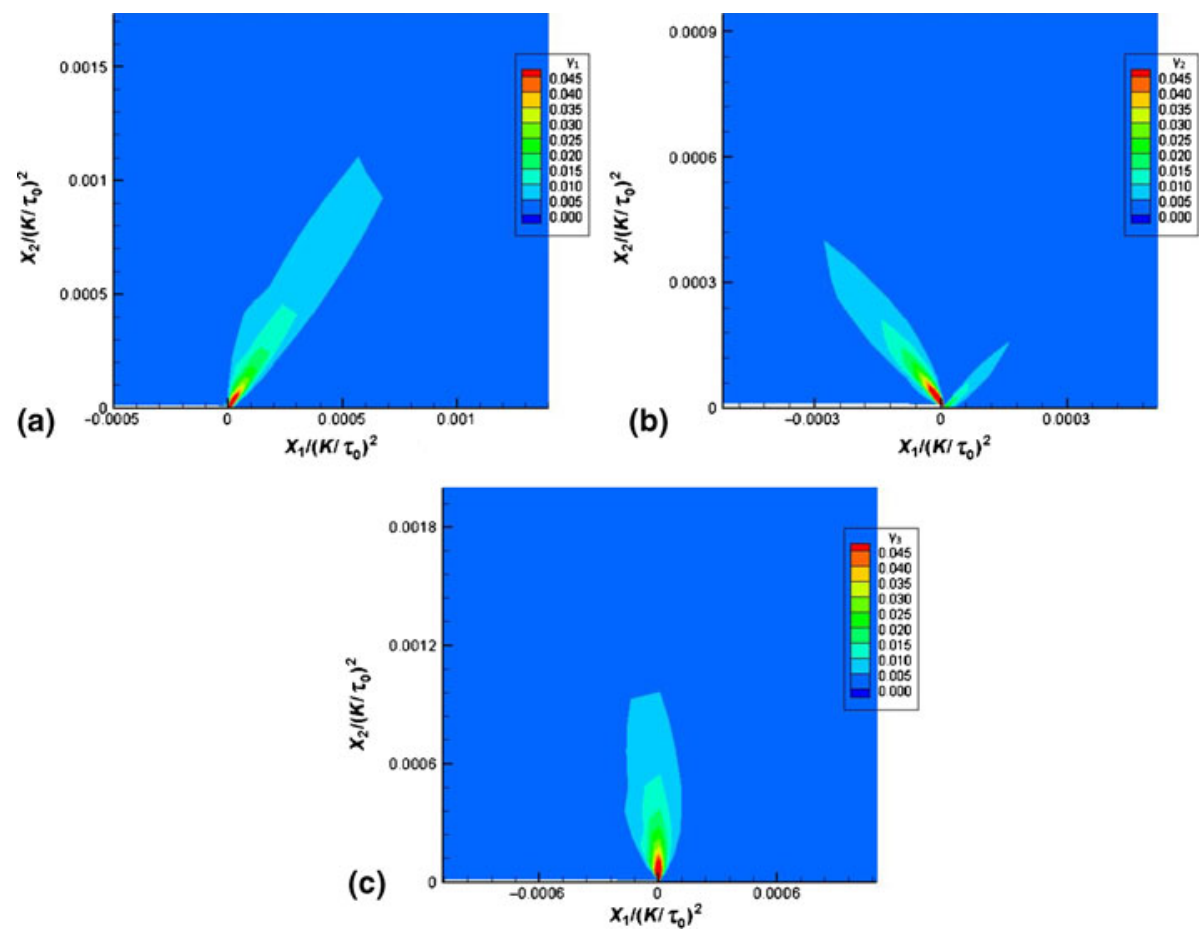

Figure 4. Fringe contours of plastic slip on slip system (a) $S_{1}$, (b) $S_{2}$ and (c) $S_{3}$ at $K=500 \mathrm{~N}-\mathrm{mm}^{-\frac{3}{2}}$, $T=0$ and $\dot{K}=10^{6} \mathrm{~N}-\mathrm{mm}^{-\frac{3}{2}} / \mathrm{s}$ for $m=0.1$.

$500 \mathrm{~N}-\mathrm{mm}^{-\frac{3}{2}}$ for different loading rates. In this plot, figures a, $\mathrm{b}$ and c correspond to $m=0.1$ but $\dot{K}=10^{4}, 10^{5}$ and $10^{6} \mathrm{~N}-\mathrm{mm}^{-\frac{3}{2}} / \mathrm{s}$, respectively, while figure $5 \mathrm{~d}$ pertains to $m=0.01$ and $\dot{K}=10^{4} \mathrm{~N}-\mathrm{mm}^{-\frac{3}{2}} / \mathrm{s}$. The same contour levels are displayed in all the figures for direct comparison. On examining figure 5a and c along with figures 3 and 4, it can be easily conceived that contributions from the slip in each slip system lead to the overall shape of the plastic zone. Thus, the three finger-like structure of the plastic zone shape is due to the slip shear band at $55^{\circ}$ and $125^{\circ}$ on slip systems $S_{1}$ and $S_{2}$, respectively, as well as due to the kink shear band at $\theta=90^{\circ}$ on slip system $S_{3}$. Similar to the observation made in the previous section, it can be seen that the plastic zone retains its shape for all loading rates (compare figure $5 \mathrm{a}, \mathrm{b}$ and $\mathrm{c}$ ). However, its size decreases with increase in loading rate. Thus, at a fixed value of $K=500 \mathrm{~N}-\mathrm{mm}^{-\frac{3}{2}}$ and $m=0.1$, the maximum spread of the contour corresponding to $\log \lambda_{1}^{p}=0.006$ remains along $\theta=55^{\circ}$ direction, but its radial extent is $0.0013\left(K / \tau_{o}\right)^{2}, 0.0011\left(K / \tau_{o}\right)^{2}$ and $0.0009\left(K / \tau_{o}\right)^{2}$ corresponding to $\dot{K}=10^{4}, 10^{5}$ and $10^{6} \mathrm{~N}-\mathrm{mm}^{-\frac{3}{2}} / \mathrm{s}$, respectively (see figure $5 \mathrm{a}, \mathrm{b}$ and c). However, Jayadevan et al (2002b) found that for an isotropic rate-dependent material obeying the von Mises yield condition, the plastic zone size is independent of loading rate. On comparing figure 5a and $\mathrm{d}$, it can be perceived that $m$ value has similar effect on plastic zone as loading rate. For example, the contour pertaining to $\log \lambda_{1}^{p}=0.006$ which spreads up to $0.0013\left(K / \tau_{o}\right)^{2}$ in figure $5 \mathrm{a}$ for $\mathrm{m}=0.1$, extends to a much larger distance of $0.0019\left(K / \tau_{o}\right)^{2}$ in figure $5 \mathrm{~d}$ for $m=0.01$. 

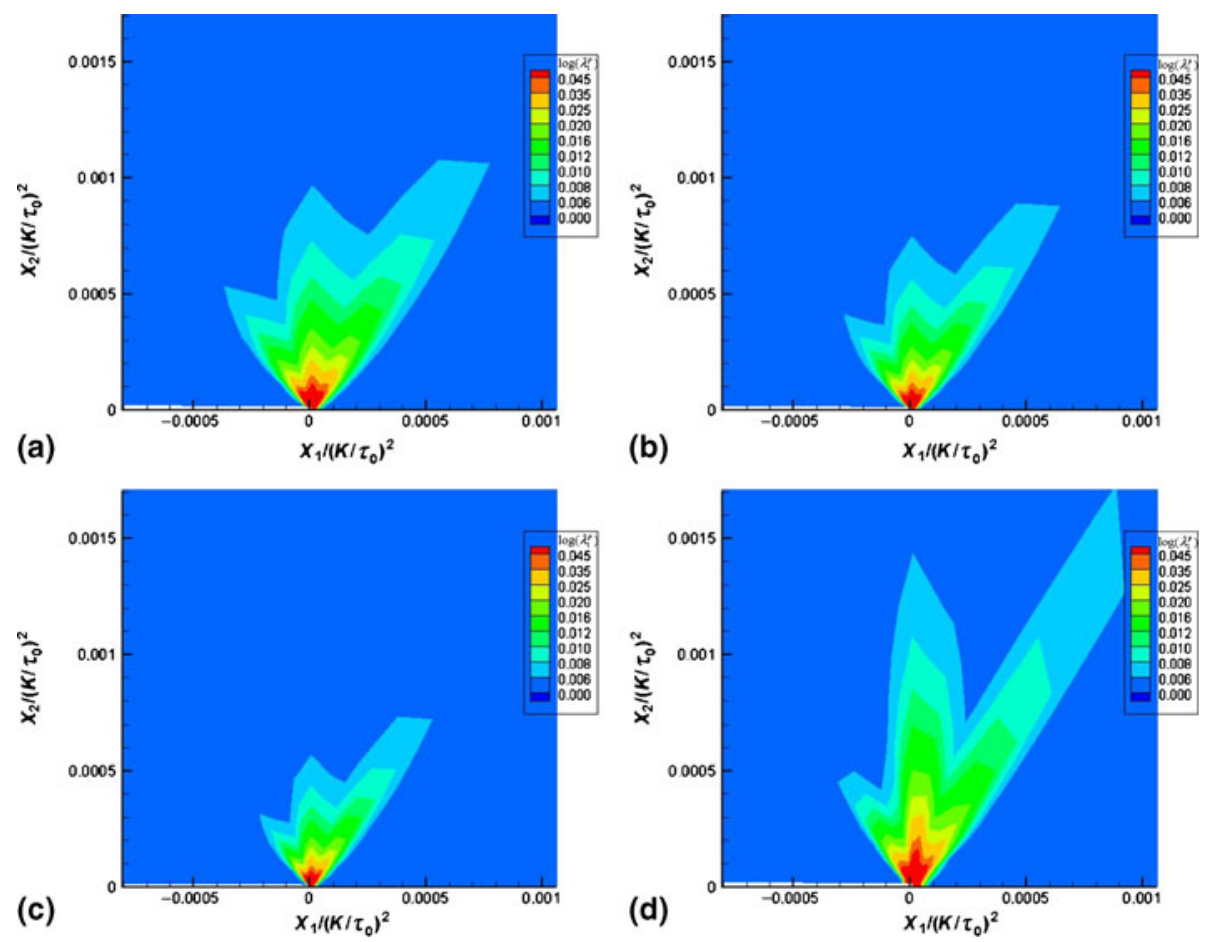

Figure 5. Fringe contours of $\log \lambda_{1}^{p}$ at $K=500 \mathrm{~N}-\mathrm{mm}^{-\frac{3}{2}}$ and $T=0$. Figure (a), (b) and (c) correspond to $m=0.1$ but $\dot{K}=10^{4}, 10^{5}$ and $10^{6} \mathrm{~N}-\mathrm{mm}^{-\frac{3}{2}} / \mathrm{s}$, respectively, while figure (d) pertains to $m=0.01$ and $\dot{K}=10^{4} \mathrm{~N}-\mathrm{mm}^{-\frac{3}{2}} / \mathrm{s}$.

The reduction in plastic zone size under higher loading rate for rate-sensitive solids can retard ductile fracture mechanisms such as void growth and coalescence. On the other hand, as already noted, enhanced stress levels at higher loading rate will accelerate the brittle fracture process. This implies that a ductile to brittle transition will happen in rate-sensitive ductile single crystals as loading rate increases. However, it must be mentioned that when inertial effect is taken into account, it may tend to decrease the stress levels at high loading rates (owing to constraint loss), as observed recently by Biswas \& Narasimhan (2011), which may counteract the above noted influence of strain rate sensitivity.

\subsection{Structure of crack-tip field}

For rate-independent single crystals, the stress field ahead of a crack tip under small scale yielding conditions can be expressed as (Patil et al 2008b):

$$
\frac{\sigma_{i j}}{\tau_{o}}=\tilde{\sigma}_{i j}\left(\frac{r}{J / \tau_{o}}, \theta, \frac{T}{\tau_{o}}, \frac{E}{\tau_{o}}, \text { Hardening parameters }\right),
$$

where, $r$ is the radial distance from the crack tip, $\theta$ is the angle measured from the crack line and $E, \tau_{o}$ are Young's modulus and initial value of critical resolved shear stress. While the stress field 
shown in figure $2 \mathrm{a}$ corresponds to this form, the stress variation in figure $2 \mathrm{~b}$ shows additional dependence on $\dot{K}$. Based on dimensional consideration and motivated by the work of Jayadevan et al (2002b) for rate-sensitive isotropic plastic solids, the structure of the near-tip stress field is postulated to have the following form:

$$
\frac{\sigma_{i j}}{\tau_{o}}=\tilde{\sigma}_{i j}\left(\frac{r}{J / \tau_{o}}, \theta, \frac{\dot{K}}{K \dot{\gamma}_{o}}, \frac{T}{\tau_{o}}, \frac{E}{\tau_{o}}, m \text {, Hardening parameters }\right) .
$$

The above implies that for a given material and fixed $T / \tau_{o}$, the parameter $\dot{K} /\left(K \dot{\gamma}_{o}\right)$ will characterize the crack tip field when the distance $r$ from the crack tip is normalized by $J / \tau_{o}$. In order to verify this self-similar structure of the crack tip stress field, radial variations of normalized stress components $\sigma_{11} / \tau_{o}$ and $\sigma_{22} / \tau_{o}$ corresponding to different combinations of $K$ and $\dot{K}$ (but with the same ratio of $\left.\dot{K} /\left(K \dot{\gamma}_{o}\right)\right)$ are presented in figure $6 \mathrm{a}$ and b, respectively, for the case $m=0.1$ and $T=0$. It can be seen from these figures that both the stress components become invariant corresponding to a fixed value of $\dot{K} /\left(K \dot{\gamma}_{o}\right)$ when plotted with respect to $r /\left(J / \tau_{o}\right)$. This confirms the validity of the form given by Eq. (6) above.

Furthermore, a reasonable form for the near-tip stress variation which explicitly reflects the dependence on the parameter $\dot{K} /\left(K \dot{\gamma}_{o}\right)$ is

$$
\frac{\sigma_{i j}}{\tau_{o}}=\left(\frac{\dot{K}}{K \dot{\gamma}_{o}}\right)^{m} \hat{\sigma}_{i j}\left(\frac{r}{J / \tau_{o}}, \theta, \frac{T}{\tau_{o}}, \frac{E}{\tau_{o}}, m \text {, Hardening parameters }\right) .
$$
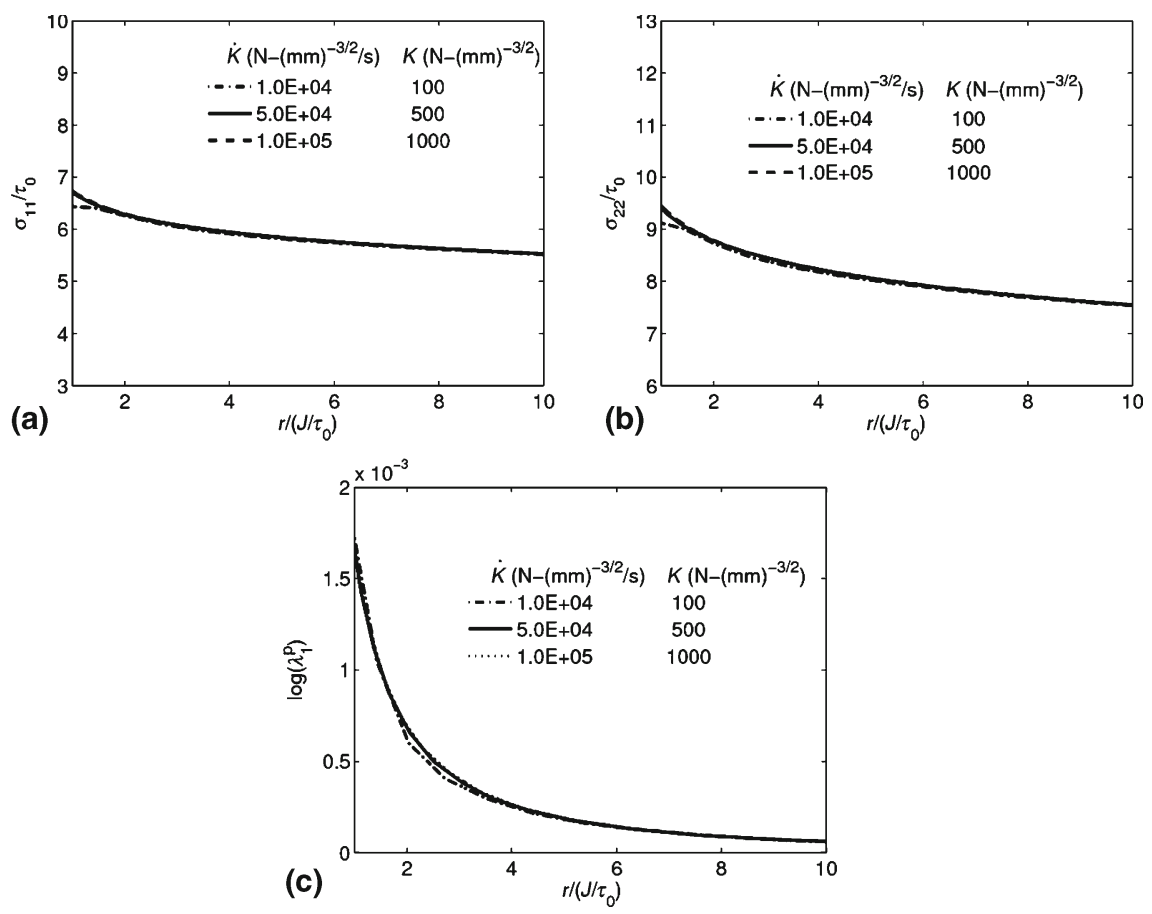

Figure 6. Radial variation of (a) $\sigma_{11} / \tau_{o}$, (b) $\sigma_{22} / \tau_{o}$ and (c) $\log \lambda_{1}^{p}$ with normalized distance ahead of crack tip for $m=0.1$ and $T=0$ at different $K$ and $\dot{K}$ but with same $\dot{K} / K$ ratio. 
The non-dimensional function $\hat{\sigma}_{i j}$ along $\theta=0$ for the case of $m=0.1$ and $T=0$ may be deduced from the plots displayed in figure 6 . In order to check the validity of the form suggested in Eq. (7), the radial variations of $\sigma_{11} / \tau_{o}$ and $\sigma_{22} / \tau_{o}$ for $m=0.1, T=0$ and $K=500 \mathrm{~N}-\mathrm{mm}^{-\frac{3}{2}}$ are presented in figure $7 \mathrm{a}$ and $\mathrm{b}$, respectively. Here, the variations obtained from finite element analysis corresponding to different $\dot{K}$ values are shown by continuous lines whereas those predicted by Eq. (7) are indicated by similar line styles along with symbols. It can be seen that the two sets of curves match well for all loading rates except at very high $\dot{K}$ of $10^{6} \mathrm{~N}-\mathrm{mm}^{-\frac{3}{2}} / \mathrm{s}$. This may be due to the fact that corresponding to this level of $\dot{K} /\left(K \dot{\gamma}_{o}\right)$ of 20000 , the plastic strain levels are low (see figure 5c) and hence the elastic-plastic stress fields may not have fully developed. Indeed, it was verified that for this loading rate, the variation obtained directly from finite element analysis converges to that predicted by Eq. (7) as $K$ increases further.

The radial variation of $\log \lambda_{1}^{p}$ is plotted in figure $6 \mathrm{c}$ for the same combinations of $K$ and $\dot{K}$ as in figure $6 \mathrm{a}$ and $\mathrm{b}$. It can be seen that similar to the radial distribution of stress components, $\log \lambda_{1}^{p}$ distribution also becomes invariant for a fixed value of parameter $\dot{K} /\left(K \dot{\gamma}_{o}\right)$ when plotted against $r /\left(J / \tau_{o}\right)$. Thus, the principal plastic logarithmic stretches also can be assumed to have the functional form given by:

$$
\log \lambda_{i}^{p}=\tilde{g}_{i}\left(\frac{r}{J / \tau_{o}}, \theta, \frac{\dot{K}}{K \dot{\gamma}_{o}}, \frac{T}{\tau_{o}}, \frac{E}{\tau_{o}}, m, \text { Hardening parameters }\right) .
$$

In the above equation, $\log \lambda_{i}^{p}$ is the $i$-th principal logarithmic plastic stretch.

Figure $8 \mathrm{a}$ and $\mathrm{b}$ show contour plots of $\log \lambda_{1}^{p}$ for $m=0.1$ corresponding to two combinations of $K$ and $\dot{K}$, but with the same ratio of $\dot{K} /\left(K \dot{\gamma}_{o}\right)$. It can be seen that the plastic zone shape and size also become identical for a given material with fixed $\dot{K} /\left(K \dot{\gamma}_{o}\right)$ when the distance is normalized by $\left(K / \tau_{o}\right)^{2}$ or equivalently by $J / \tau_{o}$. Thus, under small scale yielding conditions, the radial extent of the plastic zone at an angle $\theta$ with respect to the crack line can be expressed by the form:

$$
\frac{r_{p}}{\left(K / \tau_{o}\right)^{2}}=\tilde{h}\left(\theta, \frac{\dot{K}}{K \dot{\gamma}_{o}}, \frac{T}{\tau_{o}}, \frac{E}{\tau_{o}}, m, \text { Hardening parameters }\right) .
$$
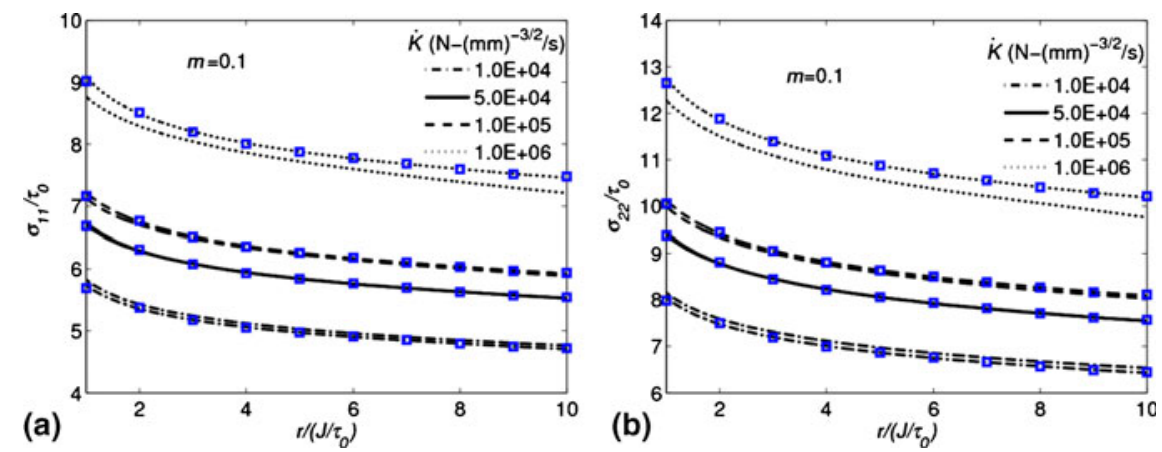

Figure 7. Radial variation of (a) $\sigma_{11} / \tau_{o}$ and (b) $\sigma_{22} / \tau_{o}$ ahead of tip obtained from scaling nondimensional function $\hat{\sigma}_{i j}$ by $\left(\dot{K} /\left(K \dot{\gamma}_{o}\right)\right)^{m}$ (see Eq. 7) along with those computed from finite element simulation for $K=500 \mathrm{~N}-\mathrm{mm}^{-\frac{3}{2}}, T=0$ and $m=0.1$. Here, the continuous solid lines represent stresses obtained from finite element analysis and those with symbols represent variations predicted by Eq. (7). 

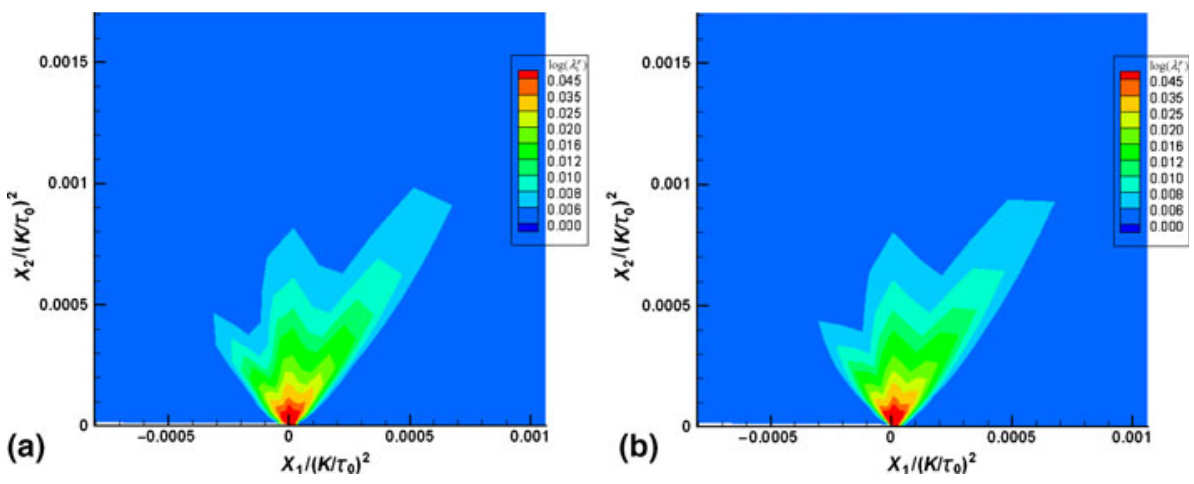

Figure 8. Fringe contours of $\log \lambda_{1}^{p}$ for $m=0.1$ and $T=0$ corresponding to constant $\dot{K} / K$ ratio with (a) $K=100 \mathrm{~N}-\mathrm{mm}^{-\frac{3}{2}}$ and $\dot{K}=10^{4} \mathrm{~N}-\mathrm{mm}^{-\frac{3}{2}} / \mathrm{s}$, (b) $K=1000 \mathrm{~N}-\mathrm{mm}^{-\frac{3}{2}}$ and $\dot{K}=10^{5} \mathrm{~N}-\mathrm{mm}^{-\frac{3}{2}} / \mathrm{s}$.

\subsection{Crack tip constraint}

In this section, the effect of loading rate and rate exponent $m$ on crack tip constraint is discussed. Attention is restricted to the radial variation of stress components ahead of the tip, plastic slip in individual slip system and overall plastic zone shape and size.

4.3a Radial variation of stress: The radial variations of opening stress component corresponding to $\dot{K}=10^{4} \mathrm{~N}-\mathrm{mm}^{-\frac{3}{2}} / \mathrm{s}$ and $10^{5} \mathrm{~N}-\mathrm{mm}^{-\frac{3}{2}} / \mathrm{s}$ are shown in figure $9 \mathrm{a}$ and $\mathrm{b}$, respectively. These plots pertain to a fixed value of $K=500 \mathrm{~N}-\mathrm{mm}^{-\frac{3}{2}}$, but at different $T / \tau_{o}$ levels. It can be seen from these figures that imposition of negative $T$-stress lowers the opening stress component considerably compared to $T=0$ case. On the other hand, positive $T$-stress marginally enhances the opening stress level. Thus, for example, at $r /\left(J / \tau_{o}\right)=4$ and corresponding to $\dot{K}=10^{4} \mathrm{~N}-\mathrm{mm}^{-\frac{3}{2}} / \mathrm{s}$ (see figure $9 \mathrm{a}$ ), the value of $\sigma_{22}$ increases to $7.5 \tau_{o}$ for $T / \tau_{o}=1$ from $7.1 \tau_{o}$ for $T / \tau_{o}=0$. At the same radial location, it drops to $6 \tau_{o}$ when $T / \tau_{o}=-1$. Further, in both the figures, the curves pertaining to different $T$-stress levels remain fairly parallel to each
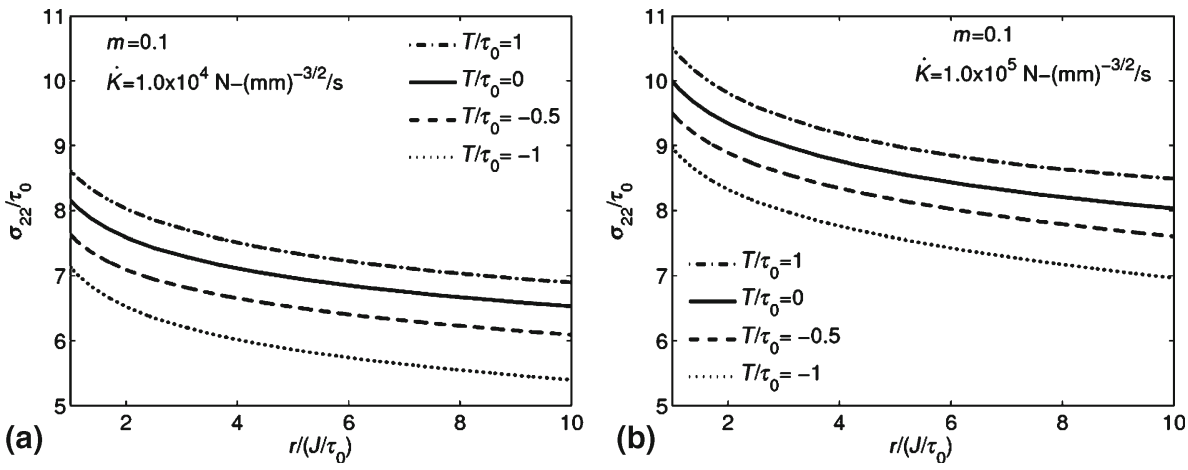

Figure 9. Radial variation of $\sigma_{22} / \tau_{o}$ ahead of crack tip with normalized distance for different $T$-stress levels corresponding to (a) $\dot{K}=10^{4} \mathrm{~N}-\mathrm{mm}^{-\frac{3}{2}} / \mathrm{s}$ and (b) $\dot{K}=10^{5} \mathrm{~N}-\mathrm{mm}^{-\frac{3}{2}} / \mathrm{s}$ at a fixed value of $K=$ $500 \mathrm{~N}-\mathrm{mm}^{-\frac{3}{2}}$. 
other for fixed $\dot{K}$. These characteristics are similar to rate-independent single crystal (Patil et al $2008 \mathrm{~b}$ ). On comparing figure $9 \mathrm{a}$ and $\mathrm{b}$ it can be observed that there is an increase in $\sigma_{22}$ stress component as $\dot{K}$ increases for a fixed $T$-stress level.

4.3b Difference stress field: One of the primary ingredients in determining the validity of twoparameter $(J-Q)$ characterization of crack-tip field, as proposed by O'Dowd \& Shih (1992), is the choice of the reference field. The choice of the reference field could be a HRR-type asymptotic solution for single crystal (see, for example Saeedvafa \& Rice 1989) or a small scale yielding solution corresponding to $T=0$. However, these solutions based on rate-independent material behaviour are not suitable to serve as the reference field for the rate-sensitive case since the loading rate strongly influences the stress distribution ahead of the tip (see figure $2 \mathrm{~b}$ ). Hence, the stress variation obtained from the small scale yielding analysis with $T=0$ corresponding to the respective rate sensitivity index $m$ and value of $\dot{K} /\left(K \dot{\gamma}_{o}\right)$ is employed as the reference solution in order to compute the difference field. This is justified in view of the forms suggested by Eqs. (6) and (7) for the near-tip stress distribution. Thus, the difference stress field components are defined as

$$
Q \hat{\sigma}_{i j}=\frac{\sigma_{i j}-\left(\sigma_{i j}\right)_{T=0}}{\tau_{o}},
$$

where $\sigma_{i j}$ and $\left(\sigma_{i j}\right)_{T=0}$ are evaluated at the same value of $\dot{K} /\left(K \dot{\gamma}_{o}\right)$.

The components of the difference stress field $Q \hat{\sigma}_{11}$ and $Q \hat{\sigma}_{22}$ are plotted against normalized distance ahead of the tip (figure $10 \mathrm{a}$ and b) corresponding to $\dot{K}=10^{4}$ and $10^{5} \mathrm{~N}-\mathrm{mm}^{-\frac{3}{2}} / \mathrm{s}$ at the same value of $K=500 \mathrm{~N}-\mathrm{mm}^{-\frac{3}{2}}$. These figures pertain to $m=0.1$. It can be seen from these figures that components of the difference field are positive when $T$ is positive and becomes highly negative as $T$ becomes negative. Thus, for example at $r /\left(J / \tau_{o}\right)=4$, the value of $Q \hat{\sigma}_{22}$ is 0.4 and -1.1 corresponding to $T / \tau_{o}=1$ and $T / \tau_{o}=-1$, respectively, for $\dot{K}=10^{4} \mathrm{~N}-\mathrm{mm}^{-\frac{3}{2}} / \mathrm{s}$ (see figure 10b). Interestingly, loading rate is seen to have only a marginal effect on the difference stress field. Further, the difference stress components remain fairly constant over the range $1 \leq$ $r /\left(J / \tau_{o}\right) \leq 10$ indicating that they are slowly varying functions of distance.

The influence of rate exponent $m$ on the radial variation of the difference stress components is shown in figure $11 \mathrm{a}$ and b corresponding to fixed $\dot{K}=10^{4} \mathrm{~N}-\mathrm{mm}^{-\frac{3}{2}} / \mathrm{s}$ and $K=500 \mathrm{~N}-\mathrm{mm}^{-\frac{3}{2}}$.
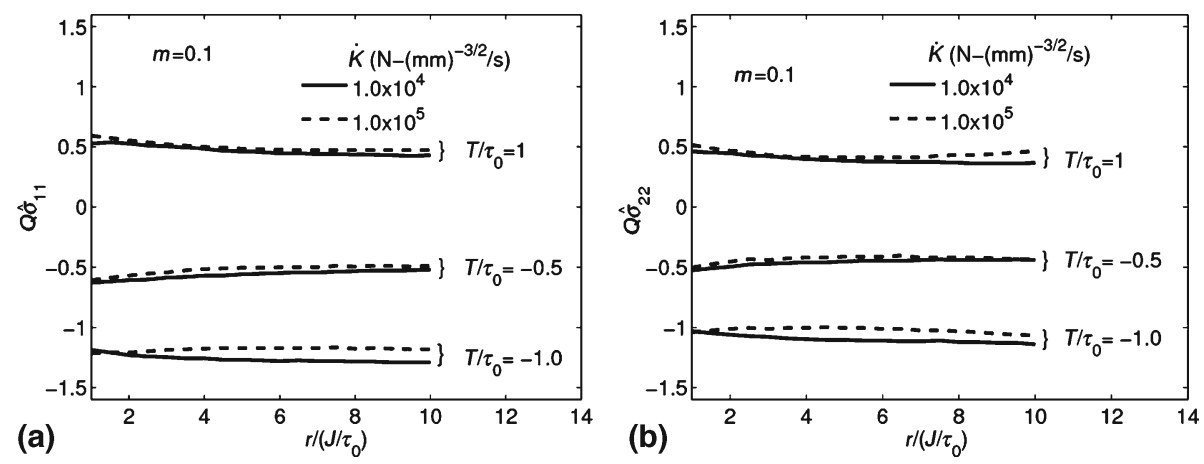

Figure 10. Radial variation of the components of difference stress field (a) $Q \hat{\sigma}_{11}$ and (b) $Q \hat{\sigma}_{22}$ with normalized distance subjected to various $T$-stress levels at $K=500 \mathrm{~N}-\mathrm{mm}^{-\frac{3}{2}}$ for two loading rates. 

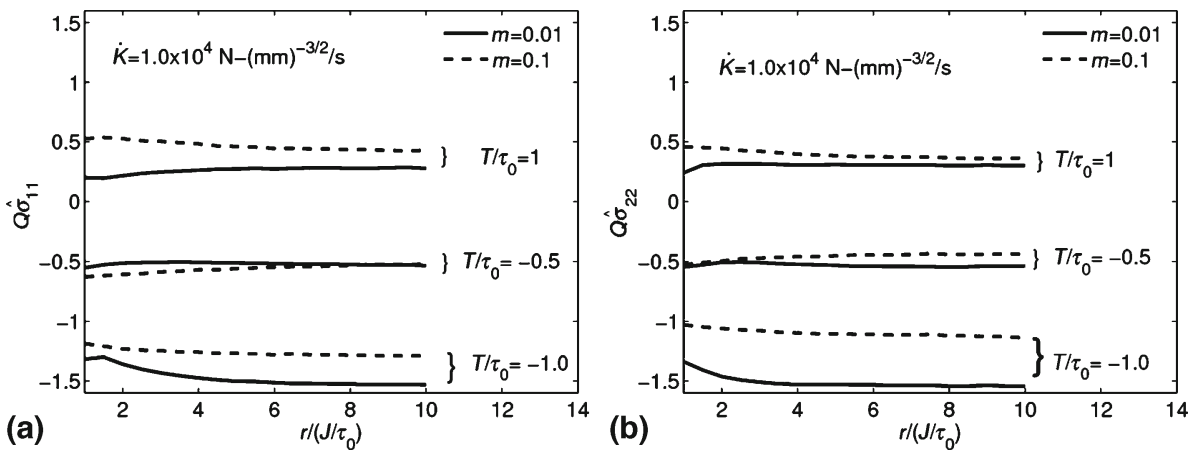

Figure 11. Radial variation of the components of difference stress field (a) $Q \hat{\sigma}_{11}$ and (b) $Q \hat{\sigma}_{22}$ with normalized distance subjected to various $T$-stress levels at a fixed value of $K=500 \mathrm{~N}-\mathrm{mm}^{-\frac{3}{2}}$ and $\dot{K}=$ $10^{4} \mathrm{~N}-\mathrm{mm}^{-\frac{3}{2}} / \mathrm{s}$ for two $m$ values.

It can be seen from these figures that the basic characteristic of the difference field remains same irrespective of the $m$ value. However, higher $m$ value shifts the curves slightly upwards indicating marginal gain in constraint when compared to lower $m$ value.

In order to quantify the difference field further, the value of $Q \hat{\sigma}_{22}$ and the ratio $Q \hat{\sigma}_{22} / Q \hat{\sigma}_{11}$ at $r /\left(J / \tau_{o}\right)=4$ are summarized in table 1 for two loading rates and three $m$ values. It can be seen that irrespective of loading rate and $m$ values, the component $Q \hat{\sigma}_{22}$ and $Q \hat{\sigma}_{11}$ changes sign with $T$-stress. This is similar to the observation made by Patil et al (2008b) for rate-independent FCC single crystal. The ratio $Q \hat{\sigma}_{22} / Q \hat{\sigma}_{11}$ remains reasonably close to unity for most of the cases. However, it was found that unlike isotropic solids (O'Dowd \& Shih 1992), the difference field is not truly hydrostatic in nature since $Q \hat{\sigma}_{33}$ is not equal to the in-plane components of the difference stress field (see also Patil et al 2008b). Thus, a constraint parameter $Q$ is defined based on the hydrostatic part of the difference stress field components as $Q=Q \hat{\sigma}_{k k} / 3$ evaluated at $r /\left(J / \tau_{o}\right)=4$. This parameter is also presented in table 1. It can be seen that $Q$ is marginally positive when $T$ is positive but it becomes strongly negative as $T$ becomes negative. Further,

Table 1. Values of different parameters associated with difference stress field for various $T / \tau_{o}, \dot{K}$ and $m$ values at a fixed value of $K=500 \mathrm{~N}-\mathrm{mm}^{-\frac{3}{2}}$.

\begin{tabular}{|c|c|c|c|c|c|c|}
\hline$m$ & $\dot{K}\left(\mathrm{~N}-\mathrm{mm}^{-\frac{3}{2}} / \mathrm{s}\right)$ & $T / \tau_{o}$ & $Q \hat{\sigma}_{22}$ & $Q \hat{\sigma}_{22} / Q \hat{\sigma}_{11}$ & $Q$ & $Q^{\prime}$ \\
\hline \multirow[t]{4}{*}{0.01} & \multirow[t]{2}{*}{$1.0 \times 10^{4}$} & 1 & 0.31 & 1.16 & 0.25 & 0.003 \\
\hline & & -1 & -1.53 & 1.04 & -1.3 & -0.018 \\
\hline & \multirow[t]{2}{*}{$1.0 \times 10^{5}$} & 1 & 0.25 & 1.17 & 0.33 & -0.004 \\
\hline & & -1 & -1.51 & 1.04 & -1.29 & 0.019 \\
\hline \multirow[t]{4}{*}{0.05} & \multirow[t]{2}{*}{$1.0 \times 10^{4}$} & 1 & 0.35 & 1.0 & 0.31 & -0.002 \\
\hline & & -1 & -1.26 & 0.91 & -1.14 & -0.004 \\
\hline & \multirow[t]{2}{*}{$1.0 \times 10^{5}$} & 1 & 0.39 & 1.02 & 0.33 & 0.004 \\
\hline & & -1 & -1.19 & 0.91 & -1.08 & -0.005 \\
\hline \multirow[t]{4}{*}{0.1} & \multirow[t]{2}{*}{$1.0 \times 10^{4}$} & 1 & 0.4 & 0.82 & 0.38 & 0.012 \\
\hline & & -1 & -1.1 & 0.87 & -1.02 & 0.008 \\
\hline & \multirow[t]{2}{*}{$1.0 \times 10^{5}$} & 1 & 0.42 & 0.84 & 0.39 & 0.008 \\
\hline & & -1 & -1.0 & 0.85 & -0.94 & -0.008 \\
\hline
\end{tabular}


comparing $Q$ values at a fixed loading rate but for different $m$ values, a small enhancement in constraint can be observed with increase in strain rate sensitivity index. Similarly, higher loading rate causes a marginal gain in constraint.

In addition, to assess the robustness of two-parameter characterization of crack tip field by $J$ and $Q$, a quantity $Q^{\prime}$ which is defined by:

$$
Q^{\prime}=\frac{\left.Q\right|_{r /\left(J / \tau_{o}\right)=8}-\left.Q\right|_{r /\left(J / \tau_{o}\right)=2}}{6}
$$

is included in table 1. Lower magnitude of the parameter $Q^{\prime}$ implies that the constraint parameter $Q$ is a slowly varying function of distance. It can be seen from the table that the parameter $Q^{\prime}$ has a very small magnitude irrespective of loading rate and $m$.

4.3c Plastic slip in individual slip systems and maximum principal plastic stretch: The contours of plastic slip in individual slip systems $S_{1}, S_{2}$ and $S_{3}$ for $m=0.1$ and $\dot{K}=10^{4} \mathrm{~N}-\mathrm{mm}^{-\frac{3}{2}} / \mathrm{s}$ at $K=500 \mathrm{~N}-\mathrm{mm}^{-\frac{3}{2}}$ are shown in figure $12 \mathrm{a}, \mathrm{b}$ and c, respectively, corresponding to $T / \tau_{o}=$ -1 . Also, contours of $\log \lambda_{1}^{p}$ are presented in figure $12 \mathrm{~d}$. A strong slip shear band which makes an angle $\theta=55^{\circ}$ with the crack line can be seen to emanate from the crack tip in figure 12a due to activity on $S_{1}$. On the other hand, activity on $S_{2}$ creates a weak but larger kink shear band at $\theta=45^{\circ}$ and a weak, small slip shear band at $\theta=125^{\circ}$ (figure 12b). Finally, activity on $S_{3}$
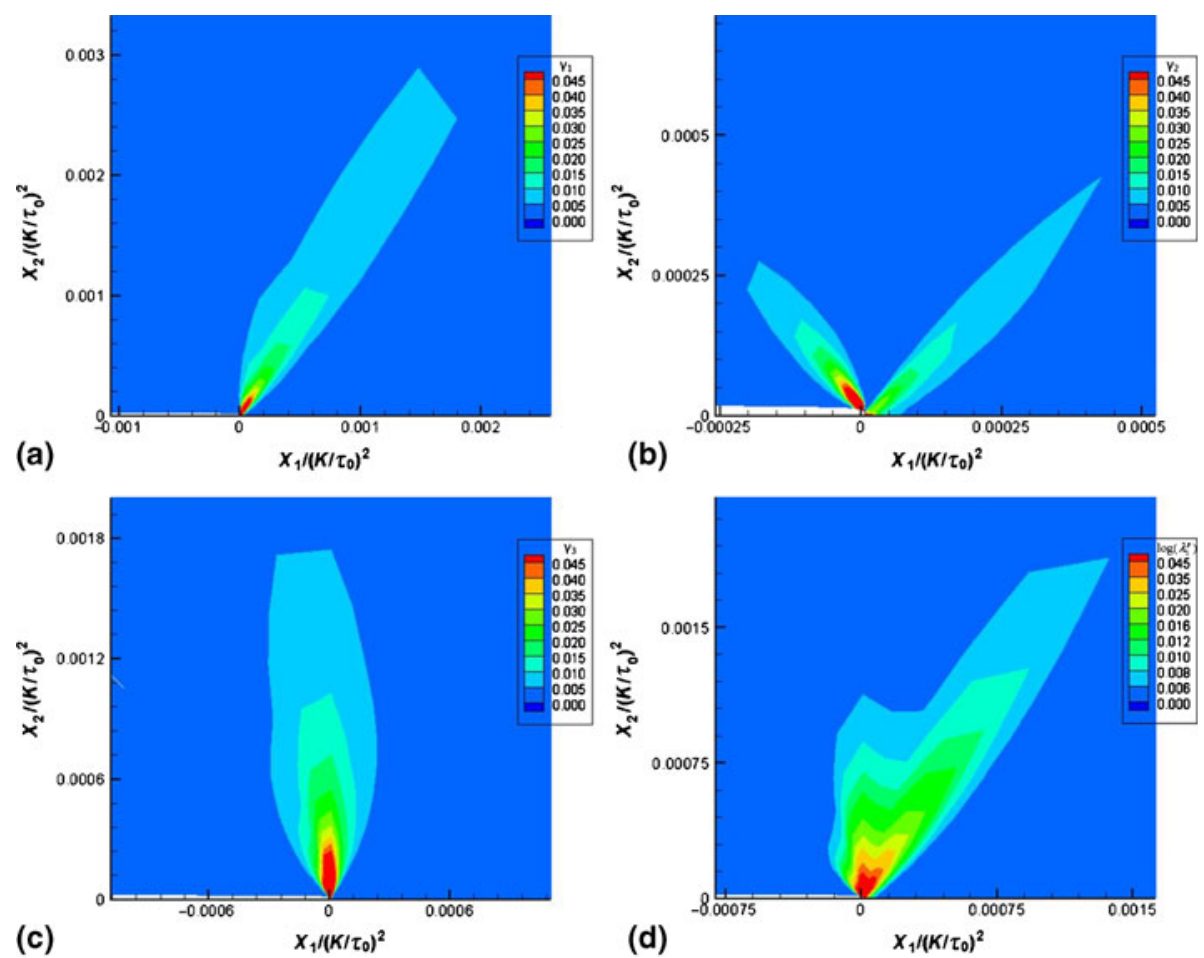

Figure 12. Fringe contours of plastic slip on slip system (a) $S_{1}$, (b) $S_{2}$, (c) $S_{3}$ and (d) contours of $\log \lambda_{1}^{p}$ at $K=500 \mathrm{~N}-\mathrm{mm}^{-\frac{3}{2}}$ and $\dot{K}=10^{4} \mathrm{~N}-\mathrm{mm}^{-\frac{3}{2}} / \mathrm{s}$ for $m=0.1$ and $T / \tau_{o}=-1$. 
produces a strong kink shear band at $\theta=90^{\circ}$ (see figure 12c). On comparing figure 12a-c with figure $3 \mathrm{a}-\mathrm{c}$, it can be seen that imposition of negative $T$-stress, increases the radial extent of the shear bands that are located in the forward sector $\left(0^{\circ} \leq \theta \leq 90^{\circ}\right)$ of the crack tip and decreases their radial extent in the backward sector $\left(90^{\circ} \leq \theta \leq 180^{\circ}\right)$. For example, the radial extent of the slip shear band at $\theta=55^{\circ}$ is $0.003\left(K / \tau_{o}\right)^{2}$ for $T / \tau_{o}=-1$ as compared to $0.0018\left(K / \tau_{o}\right)^{2}$ for $T=0$ (figure $3 \mathrm{a}$ ). On the other hand, the slip shear band at $\theta=125^{\circ}$ decreases in its radial extent from $0.0008\left(K / \tau_{o}\right)^{2}$ for $T=0$ (figure $3 \mathrm{~b}$ ) to $0.0003\left(K / \tau_{o}\right)^{2}$ for $T / \tau_{o}=-1$. Patil et al (2008b) noted similar trends for rate-independent FCC single crystal. Similarly, on comparing figures $5 \mathrm{a}$ and $12 \mathrm{~d}$, it can be noticed that the plastic zone shape for $T / \tau_{o}=-1$ is dominated mainly by the strong slip shear band at $\theta=55^{\circ}$ and does not possess the three finger-like shape seen in figure 5 a for $T=0$. From the above discussion, it follows that the T-stress may play an indirect role in changing the slip directions through the formation of kink shear bands near the tip (see, in particular, the kink shear band at $\theta=45^{\circ}$ in figure $12(\mathrm{~b})$ ).

The angular distribution of plastic slip $\gamma_{i}$ in slip system $S_{i}$ at a radial distance $r /\left(J / \tau_{o}\right)=4$ is plotted in figure $13 \mathrm{a}, \mathrm{b}$ and $\mathrm{c}$ for $m=0.1$ and $\dot{K}=10^{4} \mathrm{~N}-\mathrm{mm}^{-\frac{3}{2}} / \mathrm{s}$ at $K=500 \mathrm{~N}-\mathrm{mm}^{-\frac{3}{2}}$. Results pertaining to different $T$-stress levels are displayed. Also, the angular variation of $\log \lambda_{1}^{p}$ at the same radial distance is presented in figure $13 \mathrm{~d}$. In these plots $\theta=0^{\circ}$ and $180^{\circ}$ represent points directly ahead of the crack tip and on the crack flank, respectively. A distinct peak in $\gamma_{1}$ distribution at $\theta \approx 55^{\circ}$ can be seen from figure 13a due to the presence of strong slip shear band
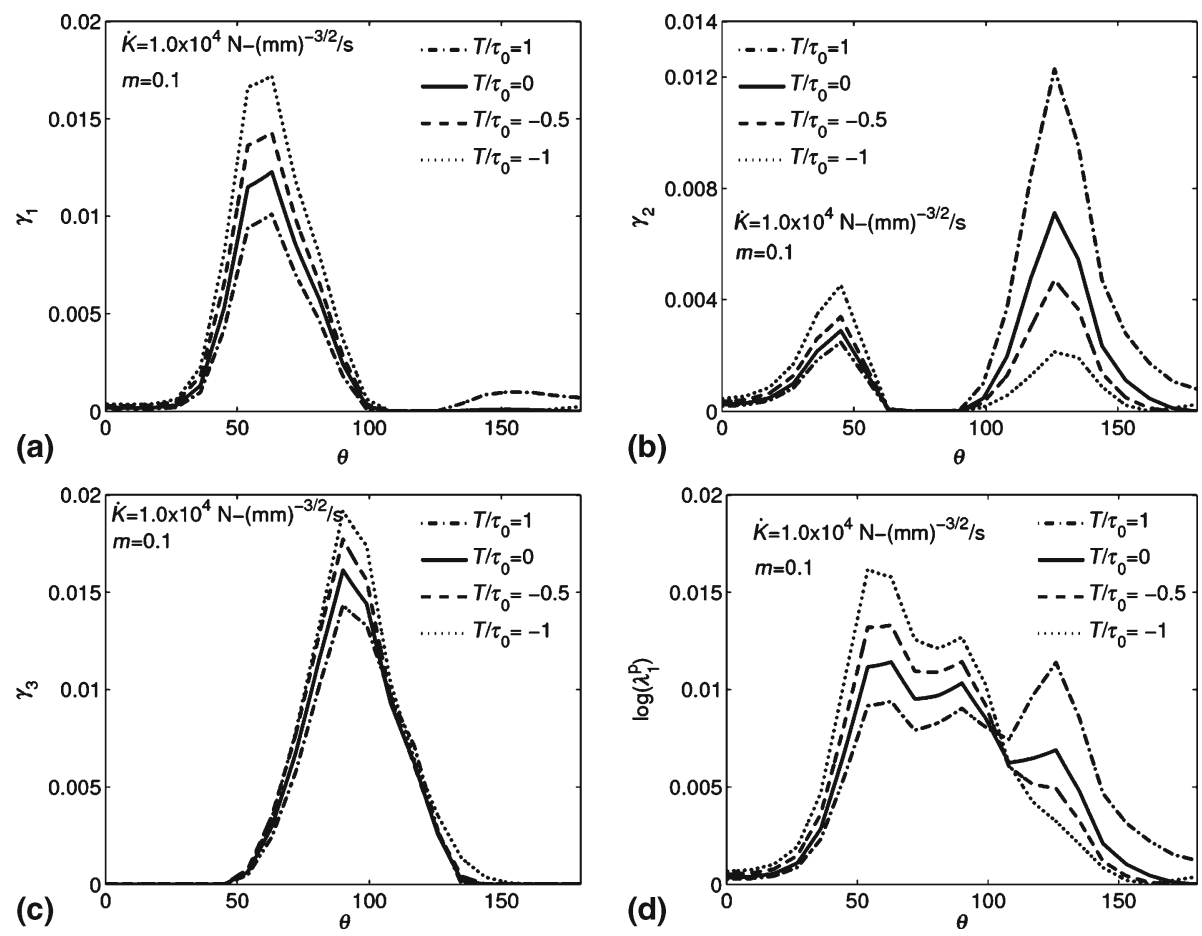

Figure 13. Angular variation of plastic slip (a) $\gamma_{1}$ on $S_{1}$, (b) $\gamma_{2}$ on $S_{2}$, (c) $\gamma_{3}$ on $S_{3}$ and (d) maximum principal $\operatorname{logarithmic}$ plastic stretch $\log \lambda_{1}^{p}$ for different $T$-stress levels at a fixed value of $K=500 \mathrm{~N}$-mm $\mathrm{mm}^{-\frac{3}{2}}$ corresponding to $\dot{K}=10^{4} \mathrm{~N}-\mathrm{mm}^{-\frac{3}{2}} / \mathrm{s}$ and $m=0.1$. 
at this angular location. The magnitude of the peak increases as $T$ becomes negative. Thus, the peak value of $\gamma_{1}$ for $T / \tau_{o}=1$ is 0.01 whereas, it is 0.017 for $T / \tau_{o}=-1$.

The angular distribution of $\gamma_{2}$ shows two distinct peaks (see figure $13 \mathrm{~b}$ ). The first peak at $\theta \approx 45^{\circ}$ is due to the kink shear band and the second peak at $\theta \approx 125^{\circ}$ is due to the slip shear band. It can be seen from this figure that as $T$ decreases, the value at the first peak increases and eventually dominates over the second peak. The second peak displays exactly opposite trend indicating the slip shear band loses its intensity as the value of $T$ decreases. On the other hand, the angular distribution of $\gamma_{3}$ (see figure 13c) shows one distinct peak at $\theta=90^{\circ}$. This peak corresponds to the kink shear band formed due to activity on $S_{3}$. The magnitude of this peak increases with decrease in $T$.

However, the angular distribution of $\log \lambda_{1}^{p}$ shows more than one peak irrespective of the level of $T$-stress (see figure 13d). The locations of these peaks for a particular $T$-stress level correspond to the peaks in individual slip systems. For, example, the first peak at $\theta=50^{\circ}-60^{\circ}$ in $\log \lambda_{1}^{p}$ distribution for $T / \tau_{o}=-1$ forms primarily due to the contribution from the strong slip shear band at $\theta=55^{\circ}$ on $S_{1}$. On the other hand, kink shear band at $\theta=90^{\circ}$ is responsible for the second peak. Further, the location of the prominent peak identifies the dominant shear band. Thus, the location of the prominent peak at $\theta \approx 55^{\circ}$ indicates that the slip shear band at $\theta=55^{\circ}$ is the dominant band for $T / \tau_{o}=-1$, wheras, it is the kink shear band at $\theta=125^{\circ}$ that dominates for $T / \tau_{o}=1$. The features discussed above agree well with the observation by Patil et al (2008b) for rate-independent single crystal.
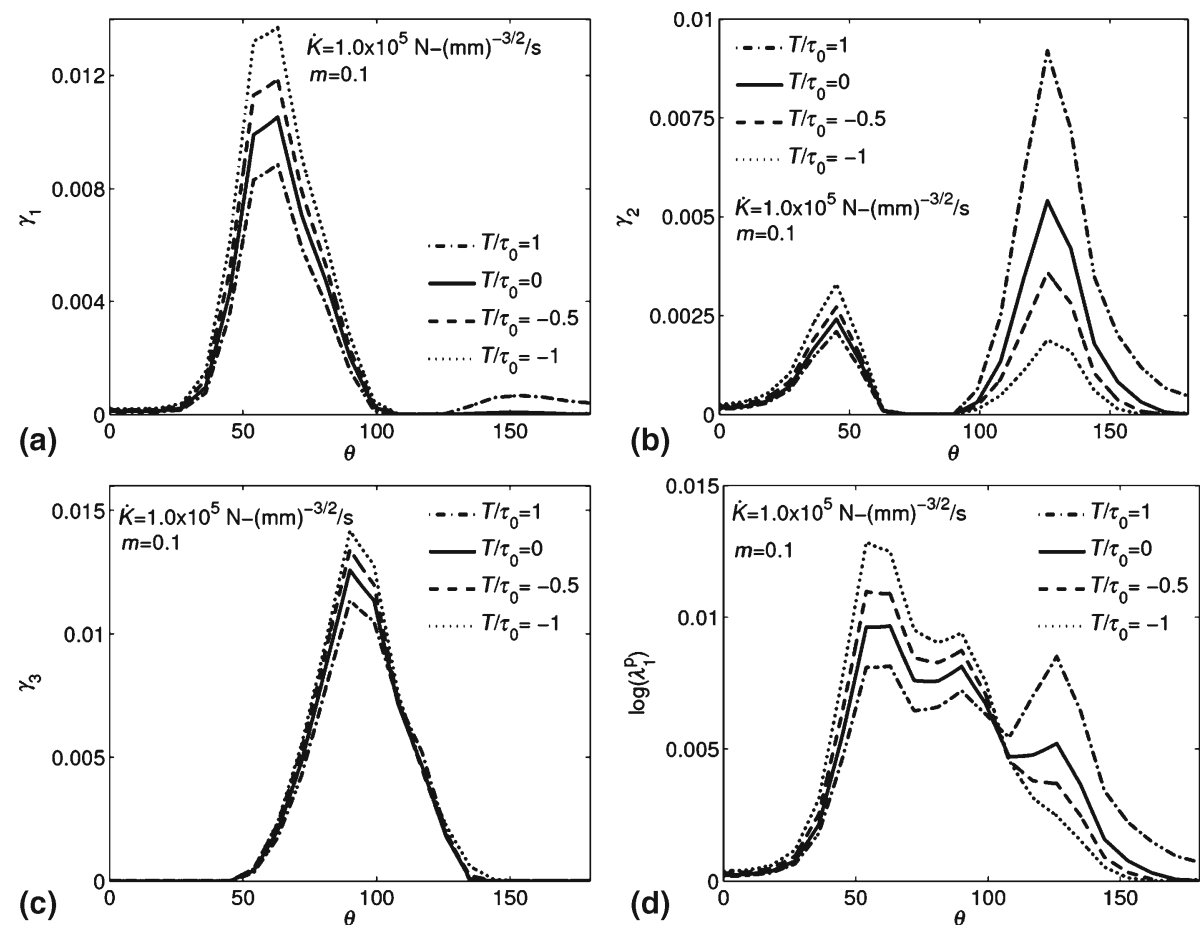

Figure 14. Angular variation of plastic slip (a) $\gamma_{1}$ on $S_{1}$, (b) $\gamma_{2}$ on $S_{2}$, (c) $\gamma_{3}$ on $S_{3}$ and (d) maximum principal logarithmic plastic stretch $\log \lambda_{1}^{p}$ for different $T$-stress levels at a fixed value of $K=500 \mathrm{~N}-\mathrm{mm}^{-\frac{3}{2}}$ corresponding to $\dot{K}=10^{5} \mathrm{~N}-\mathrm{mm}^{-\frac{3}{2}} / \mathrm{s}$ and $m=0.1$. 
Figure $14 \mathrm{a}-\mathrm{d}$ show the angular distributions of plastic slip and $\log \lambda_{1}^{p}$ at $r /\left(J / \tau_{o}\right)=4$ for higher loading rate of $\dot{K}=10^{5} \mathrm{~N}-\mathrm{mm}^{-\frac{3}{2}} / \mathrm{s}$ with $m=0.1$. In these plots, the $K$ level is same as in figure 13. On comparing figures 13 and 14, it can be seen that the effect of $T$-stress on the plastic slip remains qualitatively unaltered with change in loading rate. However, the magnitudes of the peaks reduce with increase in loading rate. Thus, the peak value of $\gamma_{1}$ corresponding to $T / \tau_{o}=-1$ is 0.017 for $\dot{K}=10^{4} \mathrm{~N}-\mathrm{mm}^{-\frac{3}{2}} / \mathrm{s}$, whereas it is 0.014 for $\dot{K}=10^{5} \mathrm{~N}-\mathrm{mm}^{-\frac{3}{2}} / \mathrm{s}$.

\section{Conclusions}

In this work, modified boundary layer simulations are conducted to investigate the role of material rate sensitivity, loading rate and constraint level on mode I crack tip fields in a ductile FCC single crystal. The following are the important conclusions of this work.

(i) At a given level of stress intensity factor $K$, the stress components ahead of the crack tip are enhanced at higher loading rate $\dot{K}$ for a rate-sensitive single crystal. On the other hand, the size of the plastic zone and magnitude of plastic slips at comparable distances from the tip are reduced at higher $\dot{K}$. The combined effect of these two trends will be to suppress ductile fracture mechanisms (like void growth and coalescence) and promote brittle mode of fracture.

(ii) It has been shown that the near-tip stress and plastic slip distributions for a given ratesensitive single crystal under small scale yielding conditions are functions of $T / \tau_{o}$ and $\dot{K} /\left(K \dot{\gamma}_{o}\right)$. The results also suggest that the stress distribution shows a simple power law scaling by the factor $\left(\dot{K} /\left(K \dot{\gamma}_{o}\right)\right)^{m}$ when the distance from the tip is normalized by $\left(J / \tau_{o}\right)$, except at very high values of the parameter $\dot{K} /\left(K \dot{\gamma}_{o}\right)$.

(iii) The stress distribution is highly sensitive to the value of $T / \tau_{o}$. There is a marginal enhancement in the direct stress components for $T / \tau_{o}>0$, whereas there is significant reduction in the stresses for $T / \tau_{o}<0$. The constraint parameter $Q$, defined in terms of the change in hydrostatic stress with reference to the $T=0$ solution (at the same level of $\dot{K} /\left(K \dot{\gamma}_{o}\right)$ ) along with $J$, is shown to provide a robust two-parameter characterization of crack tip fields in rate-sensitive single crystals, irrespective of loading rate and index $m$.

(iv) The constraint parameter $Q$ increases (i.e., there is a gain in constraint) with increase in rate sensitivity index $m$, at a given $K$ and $\dot{K}$. A similar effect of $\dot{K}$ on $Q$ for a given material (i.e., fixed $m$ ) is observed, although it is only marginal.

(v) The plastic slip shear band patterns and plastic zone shape and size depend strongly on the constraint level. Thus, negative $T / \tau_{o}$ promotes development of shear bands in the forward sector ahead of the tip and suppresses their formation behind the tip. It is important to distinguish between the effect of loading rate and that of $T / \tau_{o}$. While increase in $\dot{K}$ does not affect the qualitative nature of the shear bands and the plastic zone, but only shrinks their sizes, change in $T / \tau_{o}$ affects both the shape and size of the bands.

(vi) The operative fracture mechanism (brittle versus ductile) in rate-sensitive single crystal is expected to governed by the complex interplay of loading rate and constraint level. The latter reflects the role of fracture configuration and is known to be low for tension dominated geometries and shallow cracked bend geometries. Also, a recent study by Biswas \& Narasimhan (2011) for rate-independent single crystals and earlier works by Basu \& Narasimhan (2000), Jayadevan et al (2002a) and Biswas \& Narasimhan (2002) for isotropic plastic solids have shown that the material inertia can promote constraint loss at high loading rates even for a specimen such as a deeply cracked bend specimen which exhibits high 
constraint under static loading. The contrasting effects of rate sensitivity and the inertiadriven constraint loss will govern the operative failure mechanism and the dependence of fracture toughness on loading rate in rate-sensitive single crystals. This issue needs to be studied in a future investigation.

The authors would like to thank General Motors Research and Development Centre for financial support through project SID/IISc/GM/PC20037. The second author would also like to thank the Department of Science and Technology (Government of India) for financial support under the J.C. Bose Fellowship scheme.

\section{References}

Anderson T L 2005 Fracture mechanics: Fundamentals and applications, 3rd ed. CRC, USA

Arakere N K, Siddiqui S, Magnan S, Ebrahimi F and Forero L 2005 Investigation of three-dimensional stress fields and slip systems for FCC single-crystal superalloy notched specimens. J. Eng. Gas Turbine Power 127: 629-637

Ararkere N K, Siddiqui S, Ebrahimi F and Forero L 2009 Evolution of plasticity in notched Ni-base superalloy single crystals. Int. J. Solids Struct. 46: 3027-3044

Asaro R 1983 Mechanics of crystals and polycrystals. Adv. Appl. Mech. 23: 1-115

Basu S and Narasimhan R 2000 A numerical investigation of loss of crack tip constraint in a dynamically loaded ductile specimen. J. Mech. Phys. Solids 48: 1967-1985

Betegon C and Hancock J W 1991 Two-parameter characterization of elastic-plastic crack tip fields. Trans. ASME J. Appl. Mech. 58: 104-110

Biswas P and Narasimhan R 2002 A numerical study of constraint effects on dynamic ductile crack initiation. Mech. Mater 34(9): 577-92

Biswas P and Narasimhan R 2011 Effect of loading rate on crack tip fields in three point bend fracture specimen of FCC single. Int. J. Solids Struct. 48: 2432-2445

Blochwitz C, Jacob S and Tirschler W 2008 Grain orientation effects on the growth of short fatigue cracks in austenitic stainless steel. Mater. Sci. Eng. A 496: 59-66

Crone W C, Shield T W, Creuziger A and Henneman B 2004 Orientation dependence of the plastic slip near notches in ductile FCC single crystals. J. Mech. Phys. Solids 52: 85-112

Cuitino A and Ortiz M 1992 Computational modeling of single crystals. Model. Simul. Mater. Sci. Eng. 1: $225-63$

Duber O, Kunkler B, Krupp U, Christ H-J and Fritzen C-P 2006 Experimental characterization and twodimensional simulation of short-crack propagation in an austenitic-ferritic duplex steel. Int. J. Fatigue 28: 983-92

Garrett G and Knott J 1975 Crystallographic fatigue crack growth in aluminum alloys. Acta Metall. 23: $841-48$

Hutchinson J W 1968 Singular behavior at the end of a tensile crack in a hardening material. J. Mech. Phys. Solids 16: 13-31

Hutchinson J W 1983 Fundamentals of the phenomenological theory of nonlinear fracture mechanics. $J$. Appl. Mech. 50: 1042-1051

Jayadevan K R, Narasimhan R, Ramamurthy T S and Dattaguru B 2002a Constraint loss under dynamic loading in rate-independent plastic solids. Int. J. Fract. 116: 141-160

Jayadevan K R, Narasimhan R, Ramamurthy T S and Dattaguru B 2002b Effect of $T$-stress and loading rate on crack initiation in rate-sensitive plastic materials. Int. J. Solids Struct. 39: 1757-1775

Koppenhoefer M F and Dodds R H 1996 Constraint effects on fracture toughness of impact-loaded, precracked charpy specimens. Nucl. Eng. Des. 162: 145-158

McMeeking R M and Parks D M 1979 On criterion for J-dominance of crack tip fields in large scale yielding. ASTM, STP 668: 175-94 
Narasimhan R, Subramanya H Y, Patil S D, Tandaiya P and Ramamurty U 2009 Stationary crack tip fields in elastic-plastic solids: An overview of recent numerical simulation. J. Phys., D, Appl. Phys. 42: 214005 (15 pp)

Neumann P 1974 New experiments concerning the slip processes at propagating fatigue cracks-I. Acta Metall. 22: 1155-1165

O'Dowd N P and Shih C F 1991 Family of crack-tip fields characterized by a triaxiality parameter-I. Structure of fields. J. Mech. Phys. Solids 39: 989-1015

O'Dowd N P and Shih C F 1992 Family of crack-tip fields characterized by a triaxiality parameter-II. Fracture application. J. Mech. Phys. Solids 40: 939-963

O'Dowd N P and Shih C F 1994 Two-parameter fracture mechanics: Theory and application. ASTM STP 1207: 21-47

Patil S D, Narasimhan R, Biswas P and Mishra R K 2008a Crack tip fields in a single edge notched aluminum single crystal specimen. ASME J. Eng. Mater. Technol. 130(2): 0210131-02101311

Patil S D, Narasimhan R and Mishra R K 2008b A numerical study of crack tip constraint in ductile single crystals. J. Mech. Phys. Solids 56: 2265-2286

Patil S D, Narasimhan R and Mishra R K 2009a Families of asymptotic tensile crack tip stress fields in elastic-perfectly plastic single crystals. Int. J. Fract. 155: 143-153

Patil S D, Narasimhan R and Mishra R K 2009b Observation of kink shear bands in an aluminum single crystal fracture specimen. Sripta Met. 61(5): 465-468

Patil S D, Narasimhan R and Mishra R 2011 Effect of lattice orientation on crack tip constraint in ductile single crystals. Fatigue Fract. Eng. Mater. Struct. 34(8): 604-618

Peirce D, Asaro R and Needleman A 1983 Material rate dependence and localized deformation in crystalline solids. Acta Metall. 31: 1951-1976

Rice J 1968 A Aath-independent integral and the approximate analysis of stress concentration by Notches and Cracks. J. Appl. Mech. 35: 379-386

Rice J 1987 Tensile crack tip fields in elastic-ideally plastic crystals. Mech. Mater. 6: 317-335

Rice J R and Rosengren G F 1968 Plane strain deformation near a crack tip in a power-law hardening material. J. Mech. Phys. Solids 16: 1-12

Ritchie R O, Knott J F and Rice J R 1973 On the relationship between crirical tensile stress and fracture toughness in mild steel. J. Mech. Phys. Solids 21: 395-410

Saeedvafa M and Rice J R 1989 Crack tip singular fields in ductile crystals with Taylor Power-law Hardening. II: Plane strain. J. Mech. Phys. Solids 37: 673-691

Thomason P F 1990 Ductile fracture of metals (Oxford: Pergamon Press)

Williams M L 1957 On the stress distribution at the base of a stationary crack. Trans. ASME J. Appl. Mech. 24: $109-14$

Zhai T, Wilkinson A J and Martin J W 2000 A crystallographic mechanism for fatigue crack propagation through grain boundaries. Acta Mater. 48: 4917-27

Zienkiewicz O C and Taylor R L 1989 The finite element method, 4th ed (UK: McGraw-Hill) 